\title{
AUTO-SHAPE ANALYSIS OF IMAGE TEXTURES IN FRACTOGRAPHY
}

\author{
HYNEK LAUSCHMANN AND IVAN NEDBAL
}

Czech Technical University, Faculty of Nuclear Sciences \& Physical Engineering, Dept. of Materials, Trojanova 13, 12000 Praha 2, Czech Republic

e-mail: lausch@kmat.fjfi.cvut.cz

(Accepted. May 29, 2002)

\begin{abstract}
The aim of this study is to estimate the velocity of fatigue crack growth (crack growth rate - CGR) from the texture in SEM images of crack surfaces. A simple and quick method is based on fitting training images as a linear combination of several small subimages selected from the images themselves. The size of basic subimages is derived from autocorrelation functions of the image in row and column direction. The selection of basic subimages is based on two indicators: "appeal" evaluating their shape content, and mutual coefficient of correlation. The method is easy to implement and quick in computations, while results of testing application are fully comparable with best ones obtained within textural fractography of fatigue failures.
\end{abstract}

Keywords: decomposition, fatigue, fractography, image texture, regression.

\section{INTRODUCTION}

Within the quantitative fractography of fatigue fractures (Beachem, 1968), the traditional source of information on the crack growth rate (CGR) is striations (Nedbal et al., 1989; 1997a,b, 2000; Siegl and Matocha, 1997). As an alternative solution of the reconstitution of fatigue crack history, textural methods are being developed (Lauschmann et al., 2001). For the application of a textural method, the mesoscopic area with SEM magnifications between macro- and micro-fractography (about $30 \div 500 \mathrm{x}$ ) is especially suitable. These low magnifications were not frequently used in the past because of the absence of measurable objects in images. Examples of typical image textures are shown in Fig. 1.

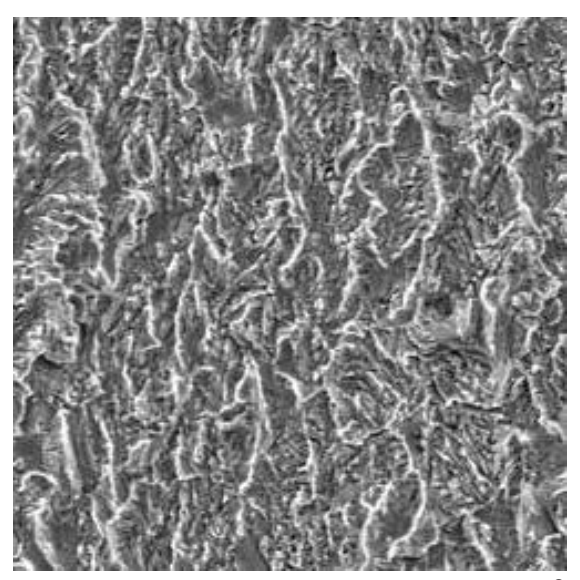

a
Three textural methods have been yet worked up to the practical application: 2D spectral analysis (Lauschmann et al., 2000), analysis of the texture as a Gibbs random field (Lauschmann and Ráček, 2001) and extraction and analysis of a fibre structure (Lauschmann, 2001). Very good results have been obtained also with 2D wavelet transform. Subsequently, other methods of textural analysis (Parker, 1997, see for an overview e.g.) are being tested.

All methods based on decomposition of the image use pre-defined basic functions. In the case of Fourier transform, the trigonometric basis is used, in wavelet transform some type of wavelets, etc. The idea of this paper is to extract the basis directly from the training images.

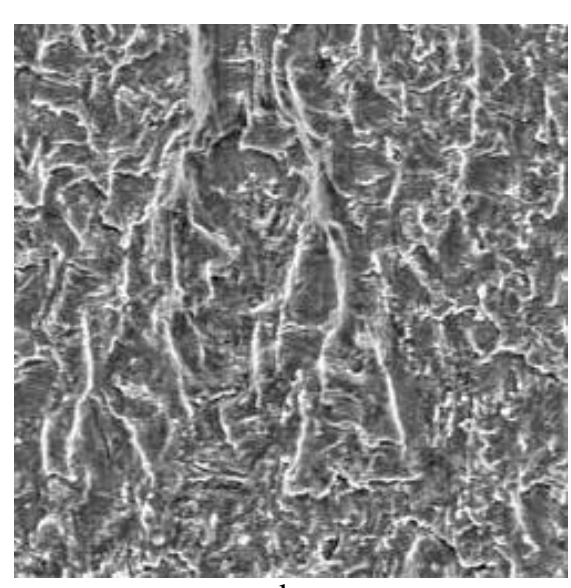

b

Fig. 1. Examples of typical textures in images of fatigue crack surface. Low (a) and high (b) crack growth rate. Stainless steel AISI 304L. 


\section{METHOD}

Methods based on decomposition of image textures into a linear combination of basic objects (planar waves, wavelets, etc.) meet a problem caused by a large amount of output data. For example, the spectral matrix contains a number of values, which equals half the number of pixels of the original image. Consequently, a special task must be solved: to analyze and express the relation between this large set of spectral coefficients, and the known value of CGR. Within the wavelet transformation, the problem is similar.

The idea of auto-shape decomposition is simple: The decomposition will be "tuned" especially for the given set of images so that the basis will not be enforced a priori but will originate from training images. Training images will be analyzed to find several special small subimages, so that the whole images could be fitted by linear combinations of them. A measure of the presence of single basic subimages within the decomposition will be taken for textural parameters. The advantage of this approach is that the output of the decomposition is a small set of coefficients, which can be simply related to the value of CGR. These coefficients reflect the occurrence of some inherent configurations of pixels in the set of images and so they are expected to reflect also the dependence on the CGR.

The set of basic subimages cannot create an orthonormal basis in a strict algebraic sense. However, this is also not necessary. The approach ought to be expressed in terms related to regression instead of decomposition. Nevertheless, to keep the continuity with usual methods, we will hold the term basic subimages, but they should be understood as a multilinear regression function.

The whole approach can be applied in the following steps:

1. The image matrix should be resized into smaller dimensions. The care should be taken of conserving the main textural structure.

2. Mean autocorrelation functions in the row and column direction of training images are computed. In both directions, the lengths of correlation, $d_{x}$ and $d_{y}$, are estimated. They should be several pixel distances. If their values are not appropriate, the ratios of resizing images (step 1) can be changed.

3. Training images are divided in a Cartesian manner into elementary subimages of the width $d_{x}$ and height $d_{y}$. Let us denote the $i$-th elementary subimage of the $j$-th image $x_{i j}$. The sequence of elementary images is not relevant. Let the number of all elementary subimages within one image be $p$. Not whole subimages around margins of the image are excluded.

4. From the whole set of elementary subimages of all training images, several ones are chosen to form the basis. The rules for selection of this set will be discussed later. A non-zero constant subimage of the same size should be added. Let us denote the set of basic subimages $\left\{b_{k}\right\}, k$ $=1,2, \ldots . n_{b}$.

5. All elementary subimages are fitted as linear combinations of the basis $\{b\}$

$$
x_{i, j}=\sum_{k=1}^{n_{b}} a_{i j k} b_{k}+\delta_{i j} .
$$

Here $\delta_{i j}$ denotes the error matrix of the same size $d_{x} \mathrm{x} \quad d_{y}$, arising from the fact that the decomposition is not total.

6. For every training image, mean absolute values of regression coefficients are computed:

$$
\alpha_{j k}=\frac{1}{p} \sum_{i=1}^{p}\left|a_{i j k}\right|
$$

7. Within the whole set of training images, the relation between CGR ( $\mathrm{CGR}_{j}$ for the $j$-th image), and the image texture, may be expressed by linear regression:

$$
C G R_{j}=c_{0}+\sum_{k=1}^{n_{b}} c_{k} \alpha_{j k}+\varepsilon_{j},
$$

where $c_{k}, k=0, \ldots . n_{b}$ are regression parameters and $\varepsilon_{j}$ denotes the residual deviation.

For a special case of analysis of textures, the simple general algorithm is completed with particular data optimized for the collection of training images: the set of basic subimages, and values of parameters $c_{k}, k=0, \ldots . n_{b}$. Within fractography, CGR may be estimated from images of fatigue crack surfaces that originated under comparable conditions as training ones. In a general texture-based classification, the set of parameters $\alpha_{j k}$ (related to the $j$-th training image and $k$-th basic subimage) can be used directly to distinguish classes of textures.

Now, we can focus on the selection of basic subimages. The frequency of occurrence of any similar brightness structures is not a suitable indicator. 
The most frequent structures do not contain shapes, being close to white noise. The intuitive requirement on basic subimages, that they should reflect typical inherent structures, may be expressed by two qualities:

- the variation of brightness within the subimage is large (it means that it contains great differences of brightness, making possible the presence of any objects on a background),

- the sum of differences between brightness in all neighbouring pixels is small (it means that pixels which are close in brightness are close in space in other words, that the image contains rather objects than noise).

To express both qualities together, an indicator called appeal was proposed. It is defined as

$$
A(x)=\frac{\operatorname{Var}(x)}{\sqrt{\sum|\operatorname{Diff}(x)|}} .
$$

Here $\operatorname{Var}(x)$ denotes the variance of brightness within image $x$ and $\sum|\operatorname{Diff}(x)|$ denotes the sum of all absolute differences between brightness in the neighbouring pixels. The form of a ratio with the square root in the denominator was found empirically by testing a number of cases.

The selection of the set of basic subimages should ensure that they are as much different as possible. Not only for the reason to generate a rich space of variability of their linear combinations, but also to get a linear system (1) that is far from being singular. It is obtained by checking mutual correlation coefficients of single basic subimages (in the sense of brightness in the corresponding pixels) while setting the basis together.

Finally, the set of basic subimages may be set in two steps:

1. Elementary subimages of all training images are sorted in decreasing order according to the value of appeal (4). The first one (with largest value) is accepted for the basis.

2. Further elementary subimages from the sequence are tested. The subimage is added to the basis, if absolute values of correlation coefficients with all subimages accepted so far are smaller than a selected limit.
With the increasing number of subimages involved in the basis, the quality of results becomes better. However, their number must not be larger than the number of pixels in the elementary subimage, $n_{b} \leq d_{x} d_{y}$.

\section{APPLICATION}

Three specimens $(\mathrm{C} 16,18)$ of stainless steel AISI 304L were loaded in the same laboratory conditions and the process of fatigue crack growth was recorded. From each fracture surface, about 45 SEM images in the magnification $200 \times$ (the field of view of $0.6 \times 0.45$ $\mathrm{mm}$ ) were obtained, and a value of the experimentally rated CGR was assigned to every image. Images were normalized (Lauschmann, 2000) to exclude large-scale fluctuations of mean brightness and contrast, by using the brightness transformation

$$
\begin{aligned}
& x_{i, j}^{\prime}=\left\{\begin{array}{cc}
0 & \text { for } u<0 \\
u \quad \text { for } 0 \leq u \leq 255 \\
255 \quad \text { for } u>255
\end{array},\right. \\
& u=128+50 * \frac{x_{i, j}-\operatorname{mean}_{S}(x)}{s t d_{S}(x)}
\end{aligned}
$$

where $\operatorname{mean}_{S}(x)$ and $\operatorname{std}_{S}(x)$ are the mean value and standard deviation of the brightness in the neighbourhood $S$ of the point $[i, j]$. The size of $S$ has been set $33 \times 33$ pixels with $[i, j]$ in the middle. As an example of application, we select training images as every second image from the set C16 (23 images).

To get a reasonably small elementary subimage, we have resized the images into the size $300 \times 400$ pixels. The autocorrelation functions for row and column directions are plotted in Fig. 2. The lengths of correlation defined by $\mid \rho_{x}\left(d>d_{x} \mid<0.05\right.$, $\mid \rho_{y}\left(d>d_{y}|<0.05|\right.$ are $d_{x}=3$ and $d_{y}=5$ pixel distances. Therefore, the dimension of elementary subimages is $5 \times 3$ pixels and images are divided into (300/5).integer $(400 / 3)=7980$ elementary subimages. The operation "integer" expresses excluding of not whole ones around margins.

The condition for selecting a subimage for the basis was set by the limit of correlation coefficients $|\rho|<0.44$. Twelve basic subimages were selected from the training images. Their position in the sequence of the largest values of appeal $A$ is evident from Fig. 3, and they are shown in Fig. 4. The basis was supplemented with a non-zero constant image to the total number of $n_{b}=13$ basic subimages. 


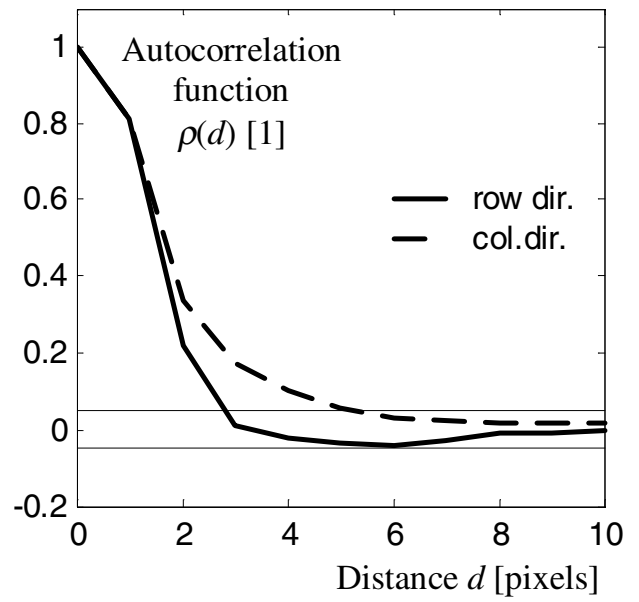

Fig. 2. Mean autocorrelation function of image brightness in row and column direction.
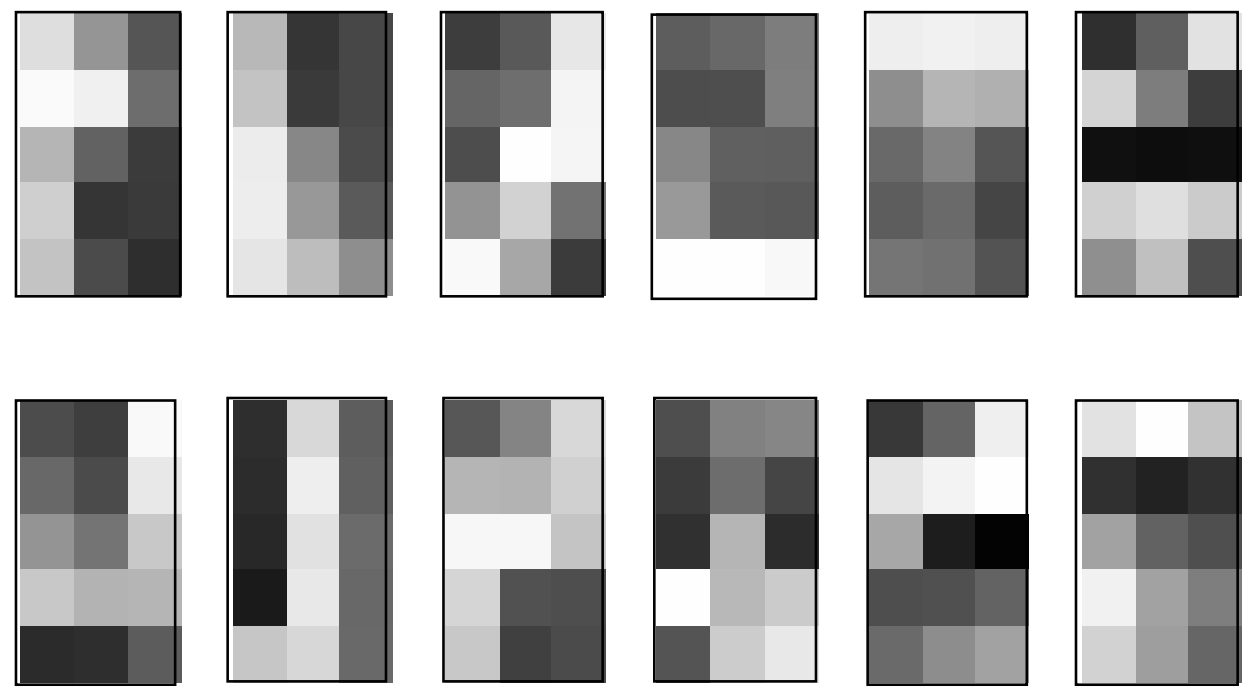

Fig. 4. Basic subimages selected from training images.

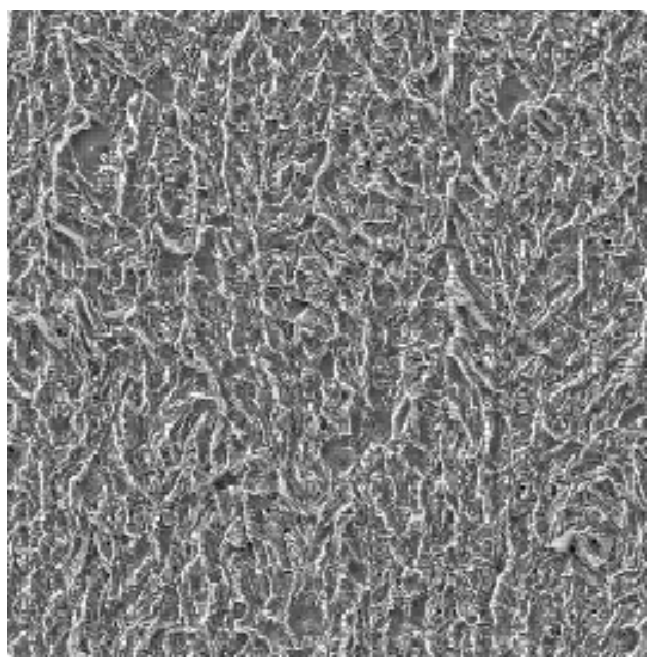

(a)

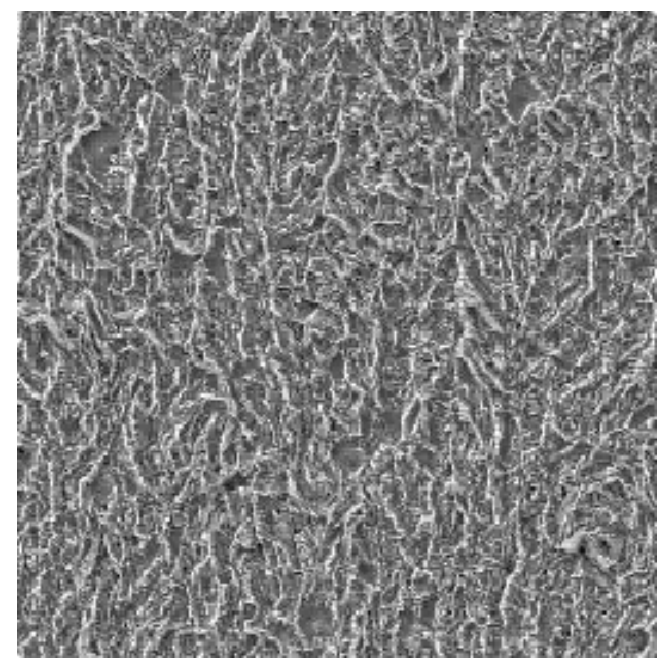

(b)

Fig. 5. Comparison of an original (a) and recomposed (b) image (sections $256 \times 256$ pixels). 
A comparison of an original image and its recomposition by elementary subimages according to (1) is shown in Fig. 5. The agreement is very good.

Final results are documented in Fig. 6. The accuracy of estimates is fully satisfactory for a practical application. The width of the strip of the estimated values of the CGR is comparable to results of textural methods that are much more sophisticated and have significantly greater requirements on computational time.

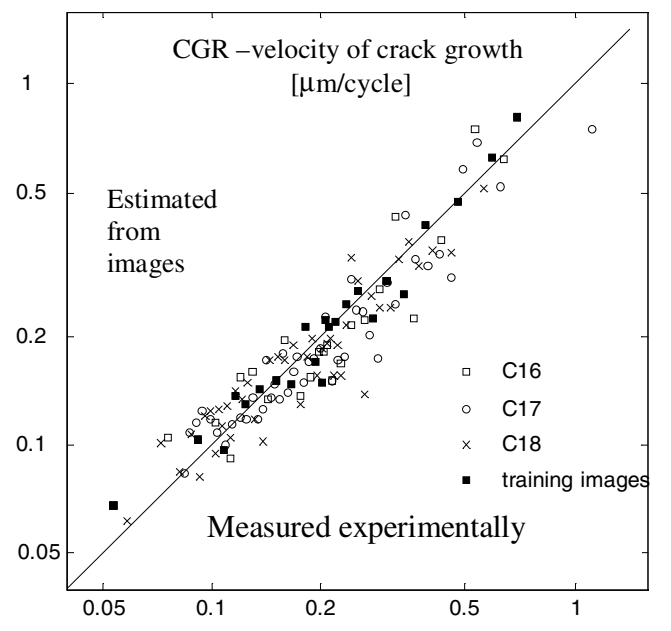

Fig. 6. Comparison of crack growth rates measured experimentally and estimated on the basis of training images. One point represents one image. The line $y=$ $x$ represents the ideal agreement.

\section{DISCUSSION}

Two indicators were chosen for the selection of basic subimages: appeal defined by the formula (4) as the measure of shape content, and mutual coefficient of correlation as the measure of independence. They were found empirically by testing many variants, as the pair giving the best results. It regards also the selection of the limit value of correlation coefficient, which generates finally the number of subimages creating the basis. To complete the set of optional parameters, the size of the mask $S$ for normalization should be mentioned. It has been set according to previous experience. Of course, we cannot say how far the empirical optimisation of the algorithm would be valid for different data sets.

The method of auto-shape decomposition is oriented entirely to the dominant component of the image texture, characterized by the largest characteristic dimensions. The information contained in smaller dimensional components of the texture (high frequencies) is more or less lost.

The division of the image into a set of elementary subimages, which are analyzed separately, introduces special limitations. The number of subimages must be large, so that they can be regarded as a random sample. It means that the dominant texture must be small in relation to the image dimensions.

\section{CONCLUSIONS}

The method of auto-shape decomposition is very simple, easy to implement and quick in computations. Results obtained are fully comparable with other textural methods used within fractography of fatigue failures, which are usually much more complicated and time-consuming.

The auto-shape decomposition performs one of ways which could be used to solve a future task of fractography: the generalisation of the traditional concept of fractographic feature into its randomized analogy - fractographic textural element. The definition of textural elements by means of basic subimages will be investigated.

From the point of view of image analysis, the core of this paper consists in its main idea. Over and above it we did not want to exceed the scope of an example. Consequently, the algorithm was optimised empirically, and its variants giving worse results were not discussed.

The basic idea is general to such a degree that it could give good results also out of frames of the textural fractography. For general application, theoretical grounds of the method should be derived, and the algorithm should be optimised generally.

\section{ACKNOWLEDGEMENT}

This research has been executed in the framework of Research project No. CEZ: J04/98: 210000021 "Diagnostics of Materials" supported by the Czech Republic.

\section{REFERENCES}

Beachem CD (1968). Microscopic fracture processes. In: Liebowitz H, ed. Fracture, An Advanced Treatise, Vol. 1. Microscopic and Macroscopic Fundamentals, 243349.

Lauschmann H (2000). Textural analysis of fatigue crack surfaces - Image pre-processing. Acta Polytechnica 40(4):123-9. 
Lauschmann H, Adámek J, Nedbal I (2000). Textural fractography: Spectral analysis of images of fatigue crack surfaces. In: Parilak L, ed. FRACTOGRAPHY 2000. (Proc. of the Int. Conf.) Košce: Institute of Materials Research of the Slovak Academy of Sciences, 313-20.

Lauschmann H, Ráček O (2001). Textural fractography: Application of Gibbs random fields. In: Proc $3^{\text {rd }}$ Int Conf Materials Structure \& Micromechanics of Fracture, Brno, June 27-29, 2001. Brno: University of Technology, 196-205.

Lauschmann H, To̊ma M, Ráček O, Nedbal I (2001). Textural fractography. Image Anal Stereol 20(S1):372-8.

Lauschmann H (2001). A database-oriented analysis of a fibre process in fractography. Image Anal Stereol 20(S1):379-85.

Nedbal I, Siegl J, Kunz J (1989). Relation between striation spacing and fatigue crack growth rate in $\mathrm{Al}$ Alloy sheets. In: Salama K et al., eds. ADVANCES IN FRACTURE RESEARCH (Proc. ICF 7, Houston) Vol. 5. Oxford: Pergamon Press, 3483-91.

Nedbal I et al. (1997a). One lecture on fractography of fatigue failures. F́ole d'f́é - Dévelopments Récents en Fatigue des Matóriaux et des Structures. SaintPierre d'Oléron, Juin 1997. Châenay Malabry: F́cole Centrale Paris - ICTM, 1-25.

Nedbal I, Kunz J, Siegl J (1997b). Microfractographic Aspects of Fatigue Crack Growth in 7010 Aluminium Alloy. In: Parilk L, ed. FRACTOGRAPHY '97. (Proc. of the Int. Conf.) Košce: Institute of Materials Research of the Slovak Academy of Sciences, 264-70.

Nedbal I, Kunz J, Siegl J (2000). Influence of corrosion environment and stress ratio on fatigue crack growth in stainless steel 304 L. In: Parilak L, ed. FRACTOGRAPHY 2000. (Proc. of the Int. Conf.) Košce: Institute of Materials Research of the Slovak Academy of Sciences, 293-300.

Parker JR (1997). Algorithms for image processing and computer vision. NewYork: John Wiley \& Sons, 150-75.

Siegl J, Matocha K (1997). Environmentally Assisted Cracking of 10GNN2MFA Low Alloy Steel. In: Parilak L, ed. FRACTOGRAPHY '97. (Proc. of the Int. Conf.) Košce: Institute of Materials Research of the Slovak Academy of Sciences, 294-9. 